\title{
Sequence variability of Rhizobiales orthologs and relationship with physico-chemical characteristics of proteins
}

\author{
Humberto Peralta, Gabriela Guerrero, Alejandro Aguilar and Jaime Mora*
}

\begin{abstract}
Background: Chromosomal orthologs can reveal the shared ancestral gene set and their evolutionary trends. Additionally, physico-chemical properties of encoded proteins could provide information about functional adaptation and ecological niche requirements.

Results: We analyzed 7080 genes (five groups of 1416 orthologs each) from Rhizobiales species (S. meliloti, R. etli, and M. loti, plant symbionts; A. tumefaciens, a plant pathogen; and B. melitensis, an animal pathogen). We evaluated their phylogenetic relationships and observed three main topologies. The first, with closer association of $R$. etli to $A$. tumefaciens; the second with R. etli closer to S. meliloti; and the third with A. tumefaciens and S. meliloti as the closest pair. This was not unusual, given the close relatedness of these three species. We calculated the synonymous (dS) and nonsynonymous (dN) substitution rates of these orthologs, and found that informational and metabolic functions showed relatively low dN rates; in contrast, genes from hypothetical functions and cellular processes showed high dN rates. An alternative measure of sequence variability, percentage of changes by species, was used to evaluate the most specific proportion of amino acid residues from alignments. When $\mathrm{dN}$ was compared with that measure a high correlation was obtained, revealing that much of evolutive information was extracted with the percentage of changes by species at the amino acid level. By analyzing the sequence variability of orthologs with a set of five properties (polarity, electrostatic charge, formation of secondary structures, molecular volume, and amino acid composition), we found that physico-chemical characteristics of proteins correlated with specific functional roles, and association of species did not follow their typical phylogeny, probably reflecting more adaptation to their life styles and niche preferences. In addition, orthologs with low dN rates had residues with more positive values of polarity, volume and electrostatic charge.
\end{abstract}

Conclusions: These findings revealed that even when orthologs perform the same function in each genomic background, their sequences reveal important evolutionary tendencies and differences related to adaptation. This article was reviewed by: Dr. Purificación López-García, Prof. Jeffrey Townsend (nominated by Dr. J. Peter Gogarten), and Ms. Olga Kamneva.

Keywords: rhizobia, comparative genomics, evolutionary rates, nonsynonymous substitution, adaptation

\section{Background}

Orthologs are genes related to a common ancestor and derived from speciation events, and their identification is often based on the most significant bidirectional similarity [1]. However, Huynen and Bork [2] proposed that chromosomal gene order conservation (gene neighbor

\footnotetext{
* Correspondence: jmora@ccg.unam.mx
Programa de Genómica Funcional de Procariotes, Centro de Ciencias

* Correspondence: jmora@ccg.unam.mx
Programa de Genómica Funcional de Procariotes, Centro de Ciencias Genómicas, Universidad Nacional Autónoma de México. Apdo. postal 565-A, Cuernavaca, Morelos, México
}

(c) 2011 Peralta et al; licensee BioMed Central Ltd. This is an Open Access article distributed under the terms of the Creative Commons Attribution License (http://creativecommons.org/licenses/by/2.0), which permits unrestricted use, distribution, and reproduction in any medium, provided the original work is properly cited.

conservation, or synteny) is also a key factor in the identification of these genes. In another study, the determination of synteny was useful to enhance the accuracy of ortholog identification in mouse and human genomes [3]. Also, we reported an analysis of chromosomal orthologs in four species of Rhizobiales, and found that syntenic genes showed more sequence conservation, higher operon organization and network formation, and encoded key metabolic proteins as compared to genes without synteny [4]. We consider that these genes can 
be viewed as a model group to study the evolutionary differences and to assess the meaning of sequence variability of orthologs.

From the dogma that genes evolve and accumulate mostly neutral mutations [5], a question arises: how to detect and evaluate if specific residues in the protein sequences encoded by orthologs are responsible for adaptation? Gene evolution can be assessed by measuring single nucleotide mutations that change (nonsynonymous, $d N$ ) or do not change (synonymous, $d S$ ) the encoded amino acid. Genes with higher nonsynonymous to synonymous ratios $(\mathrm{dN} / \mathrm{dS}>1)$ are considered to have experienced positive selection, revealing an adaptive process at the molecular level [6,7]. In bacteria, however the majority of genes evolve under purifying selection $(\mathrm{dN} / \mathrm{dS}<1)$, which is a trend that eliminates deleterious mutations. Methods in the literature to measure $\mathrm{dN}$ and $\mathrm{dS}$ rates make several inferences and use probabilistic models $[7,8]$. Evolutionary studies commonly stop there, but it is important to further study the significance of ortholog variability, considered as neutral, and trying to obtain information about how the physico-chemical properties of proteins are defined by specific sequences. We proposed that important evolutive information can be extracted directly from protein sequences through detection of the most specific residue in a given position for each species in multiple alignments of orthologs, which we represent as the percentage of change by species, originally termed as the species signature [4]. We used that measure in conjunction with the series of values reported by Atchley and collaborators [9], that consist of sets of numeric values for amino acid variability, organized by physico-chemical property (namely polarity, secondary structure, molecular volume, amino acid composition, and electrostatic charge), which summarize data from 54 amino acid attributes.

The Rhizobiales is a versatile alpha-proteobacterial Order, with members showing diversity in genomic structure, metabolic processes, and lifestyle [10-12]. The Rhizobiales include plant facultative (Agrobacterium) and animal obligate pathogens (Brucella, Bartonella), facultative symbionts (Sinorhizobium, Mesorhizobium, Rhizobium, and Bradyrhizobium) and free-living organisms (Rhodopseudomonas palustris and the plant rhizobia) [13-15]. Several complete genome sequences of Rhizobiales are available, including S. meliloti [16], $M$. loti [17], A. tumefaciens [10,12], R. etli [18], and B. melitensis [11]. Despite their described versatility, the chromosomal orthologs in these species have enough conservation of the characteristics that define the species derived from the common ancestor in the alphaproteobacterial Order [4].
As a continuation of our previous work [4], here we analyze the chromosomal orthologs of five Rhizobiales (S. meliloti, R. etli, A. tumefaciens, B. melitensis and $M$. loti) to evaluate the sequence variability among orthologs and the relationship with phylogeny and physicochemical characteristics of proteins. In this genomic study, we used the series of values of physico-chemical properties reported by Atchley et al. [9], to define how the specific properties of proteins vary according to differences in amino acid residues, and also to determine the significance of the percentage of changes by species, in relation to variability. The aim of this study were to analyze the evolution of common orthologs in rhizobiales species, to extract the specific changes in their sequences, in relation to other evolutionary measures and to assess, theoretically, the physico-chemical impact of these changes. We hypothesize that many changes in sequences are not completely neutral, but define physico-chemical differences with a role in adaptation to species niches.

\section{Methods}

\section{Detection of orthologs}

The following genome sequences of Rhizobiales species were analyzed in this work: Sinorhizobium meliloti 1021 (hereafter referred as $S m$ or $S$, Genbank accession number NC_003047), Rhizobium etli CFN42 ( $R e$ or $R$, NC_007761), $R$. etli strain CIAT652 (CIAT, NC_010994), Agrobacterium tumefaciens C58 (At or A; Cereon, NC_003062), Mesorhizobium loti MAFF303099 ( $M l$ or M, NC_002678) and Brucella melitensis 16M (Bm or B, NC_003317). These sequences were obtained from the NCBI web site (http://www.ncbi.nlm.nih.gov). Orthologs were detected by the best bidirectional hit between pairs of species, with $S$. meliloti as a reference, using the program Fasta34 [19] with the following parameters: an identity of at least $50 \%$, overlapping by at least $150 \mathrm{nt}$, and an (E) score $<10^{-5}$. Orthologs in the five chromosomes were determined by combining the results of each pairwise comparison in order to get the common set. The correct ortholog correspondence among pair comparisons was manually checked. Additionally, to get a more strict ortholog assignation, the genes must have a synteny relationship (or conserved neighborhood), and for this we applied a method reported previously [4].

\section{Functional classification of the chromosomal orthologs}

The functional annotation of the orthologs was that from A. tumefaciens C58-U. Washington annotation [10]; functions were manually checked against the other genomic functional annotations. The functions were classified into groups as follows. Metabolism: amino 
acid, nucleotide, fatty acid, and cofactor synthesis; central intermediary metabolism, and energy obtention; informational: DNA metabolism (including recombination, replication and repair), transcription, translation, and regulatory functions; processes: transport, cell envelope synthesis, and cellular processes (such as cell division, secretion and chemotaxis); and hypothetical and unknown functions.

\section{Identity, similarity and the percentage of changes by species (\%chSp)}

Similarity (pair comparisons) and common identity (multiple comparisons) were calculated with ClustalW alignments [20]. The percentage of changes by species (or \%chSp), originally the species signature, was calculated as reported previously [4], and consists, briefly, in the amount of most specific residues for each ortholog, reported as a percentage, extracted from homologous positions in ClustalW multiple alignments. The cases when a position was considered part of the $\%$ chSp were: four identical residues and one different, three identical residues and two different (\%chSp accounted for two species), two identical residues and three different (\% chSp accounted for the three species), and five different residues (\%chSp accounted for the five species). The percentage was calculated dividing by a common length. Gaps (internal differences) and non-aligned sequences (in the extremes of the sequences), were not considered and were trimmed with an ad hoc perl script, following accepted criteria.

\section{Phylogenetic analysis}

Phylogenetic trees were obtained with the neighbor joining (NJ) and maximum likelihood (ML) methods. Sequences were first aligned with the ClustalW program, and the non-aligned segments and gaps were cut with ad hoc perl scripts. Unrooted phylogenies were calculated for each of the orthologs separately. For ML, we used the proml program, and for the NJ method, the neighbor program, both from the Phylip package v3.6 [21] by Joe Felsenstein, available on the web (http:// www.evolution.genetics.washington.edu/phylip.html). The JTT model of substitution was applied. The ML and NJ methods gave similar results, and we chose to work with those obtained with ML.

\section{Synonymous and nonsynonymous substitution rates}

Estimation of the number of synonymous (dS) and nonsynonymous $(\mathrm{dN})$ substitutions per site was performed by $\mathrm{ML}$ analysis with the codeML program of the PAML package [22], used with model 1 (free ratio), runmode 0 (user tree), fixed branch length initial, and with the empirical F3 $\times 4$ nucleotide frequencies model. The amino acid sequence alignments were traced back to their corresponding nucleotide sequences and the nonhomologous segments were trimmed with an ad hoc perl script. $\mathrm{dN}$ and $\mathrm{dS}$ values corresponded to the final branch. Genes with $\mathrm{dN}$ values above 0.4 , or $\mathrm{dS}$ values above 5, were eliminated from the analysis. The orthologs were assigned to the group with low $\mathrm{dN}$ value if, according to the order of the five $\mathrm{dN}$ values of each ortholog, the second lowest value did not surpass the $0.05 \mathrm{dN}$ limit. Otherwise, the ortholog was assigned to the high $\mathrm{dN}$ group. Codon usage tables were obtained from the Kazusa website (http://www.kazusa.or.jp/ codon).

\section{Physico-chemical series of values for amino acids and prediction of transmembranal proteins}

We used the series of values for amino acids (polarity, secondary structure, molecular volume, amino acid composition, and electrostatic charge) reported by Atchley et al. [9]. Amino acid values in the series of physicochemical properties are positive or negative. To calculate the value for each sequence, the values for each residue were added, with sign conservation. Negative or positive values were obtained, with the exception of the amino acid composition, which always yields positive values. Clustering of results was performed with Multiple Experiment Viewer MeV TM4 [23], used with the normalized data from the values of physico-chemical properties, in hierarchical mode, with the Pearson rank correlation or Euclidean distance, and average linkage. To normalize values, all values in each row of data were multiplied by a scale factor $S$, so that the sum of the squares of the values in each row was 1 (a separate $S$ factor is computed for each row). To normalize array (columns), a similar computation was performed. For the relationship of the \%chSp with physico-chemical properties, the method described by Olivares-Hernandez et al. was followed [24]; briefly, range values of all properties and average of the \%chSp of all species were calculated. To obtain clusters of products with high correlation values, the range of each property was divided by the average \%chSp from the five organisms, the result was multiplied by $\log 2$ and clustered with the Multiple Experiment Viewer MeV TM4 program [23], using k-means and Euclidean distance. The TMAP program, version 46 [25], available in the EMBOSS package (http://www.emboss.org), was used to predict transmembranal segments in the protein sequences.

For functional analysis, orthologs were classified as positive or negative if values for each ortholog product from the five species had the same sign (in the case of amino acid composition, values were divided by half and assigned to low or high level groups). The resulting groups were: polarity, negative 725 sequences, positive 297; secondary structure, negative (representing 
abundance of alpha-helices) 530, positive (abundance of coils) 314; molecular volume, negative (representing products with small residues) 886 , positive (products with bulky residues) 72; amino acid composition, low (represents atypical composition) 612, high (very typical composition) 621; electrostatic charge, negative 613, positive 191.

\section{Statistical analysis}

The Mann-Whitney test was used to evaluate differences in frequency distributions and was performed with Minitab v12 (Minitab, Inc.). The Fisher's exact tests for significant differences in proportions were calculated with Oyvind Langsrud's page (http://www.langsrud.com/fisher.htm). Pearson correlation coefficients were calculated with Minitab v12.

\section{Results}

We attempted to use the analysis of ortholog sequence variability by the percentage of changes by species (\% chSp) in Rhizobiales genomes [4] as an instrument to determine genomic differences among groups of species, and evolutive coherence. This is connected with the phylogenetic relationships of the species, but also with the chemical nature of amino acid substitutions in proteins. Thus, we assessed the relationship of sequence variability with phylogeny and physico-chemical properties of a set of 1416 orthologs from five species of Rhizobiales (S. meliloti, R. etli, A. tumefaciens, B. melitensis and $M$. loti). We applied a measure we proposed, termed the percentage of changes by species, originally species signature (defined as the normalized number of residues in the ortholog sequence that occur only once in individual alignment columns) [4], intended to extract species-specific information, possibly related to the adaptation to niche, and compared the \%chSp with the evolutionary rates of the orthologs (synonymous and nonsynonymous substitution rates). Additionally, we used five series of values for amino acid properties (polarity, secondary structure formation, volume, amino acid composition and electrostatic charge) reported by Atchley et al. [9], to explore the relationships of physico-chemical properties of residues encoded in the orthologs with phylogeny, evolutionary rates and function.

\section{The percentage of changes by species (\%chSp) and relationships with function and $\mathrm{dN}$ and $\mathrm{dS}$}

With the \%chSp we wanted to find the specific differences in protein sequences of the orthologs for each of the species studied. The \%chSp was calculated by normalizing the number of residues in the ortholog sequence that occur only once in individual alignment columns [4]. This measure denotes the amount of most specific residues in the orthologs, and is expressed as a percentage of the total number of amino acids in the proteins. In Figure 1A, the \%chSp of the orthologs studied is shown. Two groups can be clearly distinguished, with S. meliloti grouped with A. tumefaciens and R. etli, and $M$. loti and B. melitensis in another group. That grouping reflects the phylogenetic relationship of these species. Frequency distribution curves of the \%chSp values were plotted to illustrate in detail the separation of these groups (Figure 1B).

To detect the effect of divergence on functional role, we plotted the distribution of functional classes by the \%chSp. As observed in Figure 1C (for R. etli, the other species gave a similar pattern, not shown), the informational functions (replication and DNA metabolism, transcription, translation, and transcriptional regulation) showed the most reduced amount of the \%chSp, followed by metabolic functions, and cellular processes. The hypothetical functions or poorly characterized proteins showed the highest level.

To determine the usefulness of the \%chSp, we compared this parameter with the codon evolution of the orthologs. To do this, we calculated the synonymous $(\mathrm{dS})$ and nonsynonymous $(\mathrm{dN})$ substitution rates by the maximum likelihood method with PAML [22]. Some genes showed $\mathrm{dS}$ saturation and were eliminated from the analysis; 985 genes remained with $\mathrm{dS}$ values lower than 5 . We obtained very high correlations between the $\% \mathrm{chSp}$ and $\mathrm{dN}$ (Figure 2A) and dS (Figure 2B), with Pearson coefficients of $r=0.932$ to 0.953 (with $p<0.05$ ) for relation with $\mathrm{dN}$, and $\mathrm{r}=0.517$ to $0.587(\mathrm{p}<0.05)$ for dS. These data revealed that the $\%$ chSp was well correlated with codon evolution and is a useful tool to extract directly the evolutive change at the protein level. In comparison, other measures of sequence conservation, such as protein identity or similarity, gave lower Pearson coefficients with dN or dS (for example, in $S$. meliloti, $\mathrm{dN}$-common identity $\mathrm{r}=-0.798, \mathrm{dN}$-common similarity $\mathrm{r}=-0.650$; $\mathrm{dS}$-common identity $\mathrm{r}=-0.511$, $\mathrm{dS}$-common similarity $\mathrm{r}=-0.400$ ) (not shown). $\mathrm{dN}$ and the $\%$ chSp are related measures, but they are not derived from the same sequence information: $\mathrm{dN}$ is calculated from nonsynonymous codons in nucleotide sequences, while the \%chSp is calculated directly from protein sequences by extracting the most specific residue for each homologous position after sequence alignment.

Based on values obtained from each of the five species (see Methods section), the orthologs were classified into groups either with low or high dN: 426 were classified as having a low $\mathrm{dN}$ value, and 559 as having a high $\mathrm{dN}$ value. A functional distribution was generated and, significantly, metabolism (amino acid and nucleotide biosynthesis and energy generation), and informational 

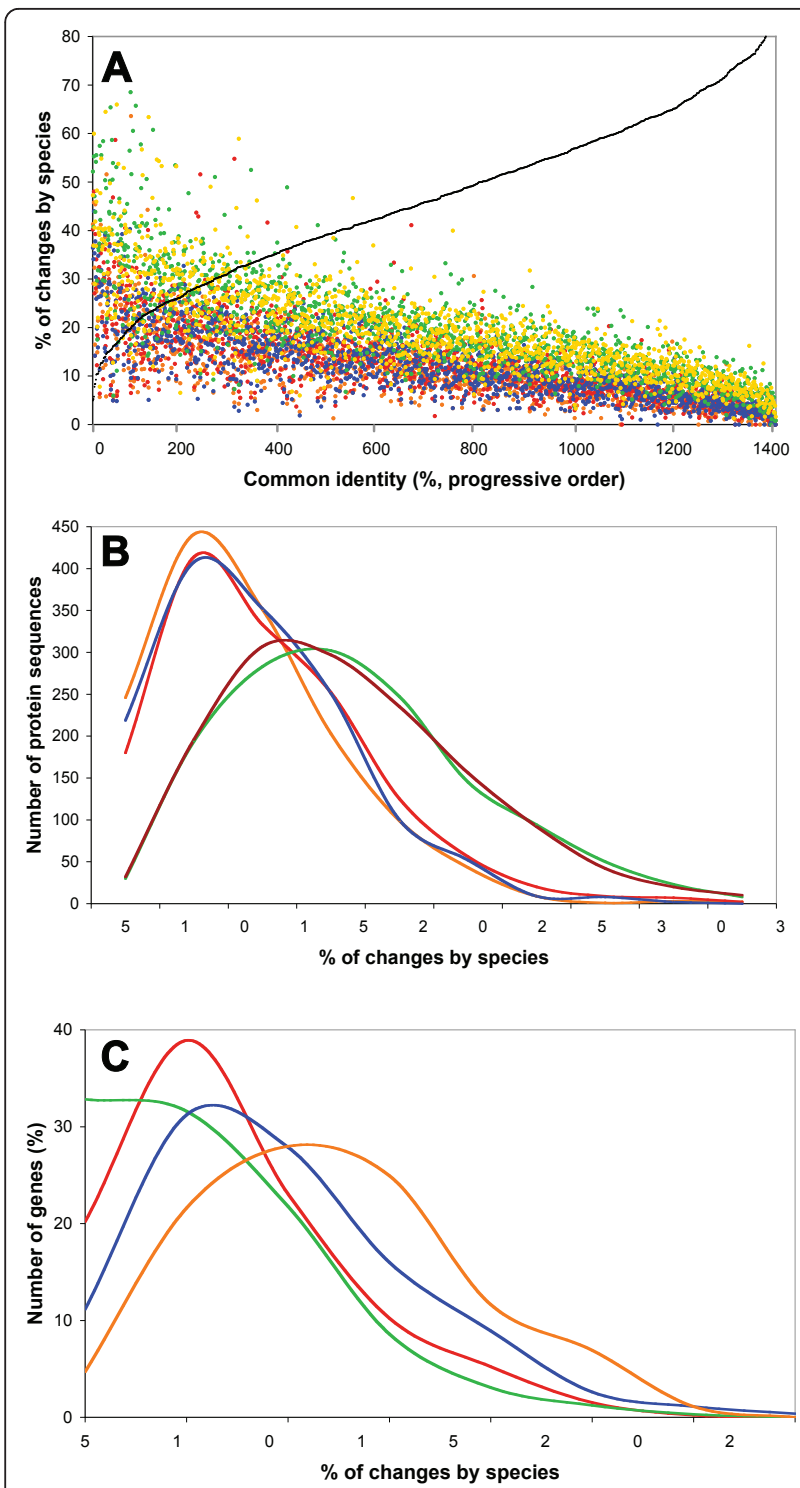

Figure 1 The percentage of changes by species (\%chSp) of 1416 chromosomal orthologs from five Rhizobiales. The panels show 1416 orthologs for each of the species (7080 ortholog products in total). A, the \%chSp arranged progressively according to the $\%$ of common identity of each ortholog from the five organisms (black line). Color dots: orange, R. etli; red, A. tumefaciens; blue, S. meliloti; green, Brucella melitensis; and yellow (or brown) M. loti. B, frequency distribution curves for the \%chSp of the five organisms. Values above $50 \%$ were omitted for clarity. Color lines as in $\mathrm{A}$, with exception of brown for M. loti. C, functional distribution of orthologs by species signature. Data from $R$. etli products. Functions were classified into Metabolism (red line, including amino acid, nucleotide, fatty acid, and cofactor biosynthesis, central intermediary metabolism, and energy generation), Information (green line, DNA metabolism, transcription, translation, and transcriptional regulators), Processes (blue line, transport; cellular envelope synthesis, and other cellular processes), and Hypothetical functions or poorly characterized proteins (orange line).
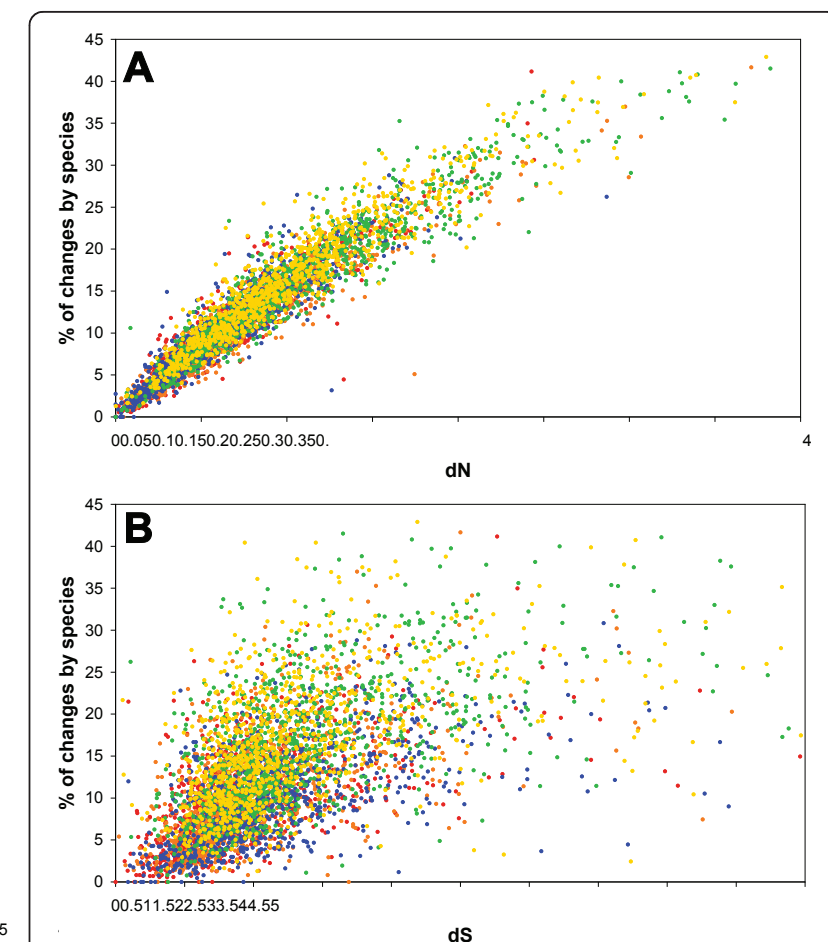

Figure 2 Relationship of the percentage of changes by species (\%chSp) of orthologs with synonymous (dS) and nonsynonymous $(d N)$ substitution rates. $A$, relationship of $d N$ with the \%chSp. B, relationship of dS with the \%chSp. dN and dS values were obtained with PAML [22]. Dot colors: orange, R. etli; red, A. tumefaciens; blue, S. meliloti; green, B. melitensis; and yellow, M. loti.

(transcription and translation) functions tended to be encoded by genes with low $\mathrm{dN}$ values (Figure 3 , significant difference by Fisher's exact test, with $\mathrm{p}<0.05$ ). In contrast, genes belonging to the high $\mathrm{dN}$ group had signifícant abundance of hypothetical functions, and also contributed particularly to cellular processes (transport and cellular envelope). The significance of these differences is explained in the Discussion section.

\section{Phylogenetic relationships of orthologs}

To detect the phylogenetic relationships in the orthologs under study, we obtained their phylogenetic trees by maximum likelihood with Phylip [21]. Three main topologies were found. The first, which included $52 \%$ of the orthologs, associated $R$. etli with A. tumefaciens in the same branch (denoted as $R$. etli-A. tumefaciens, $S$. meliloti, Brucella melitensis-Mesorhizobium loti or a RA-S-BM topology, for the initials of the species); the second, comprising $24 \%$, joined R. etli with S. meliloti (RS-A-BM topology); and the third, with 13\%, connected A. tumefaciens with S. meliloti (AS-R-BM topology). The 


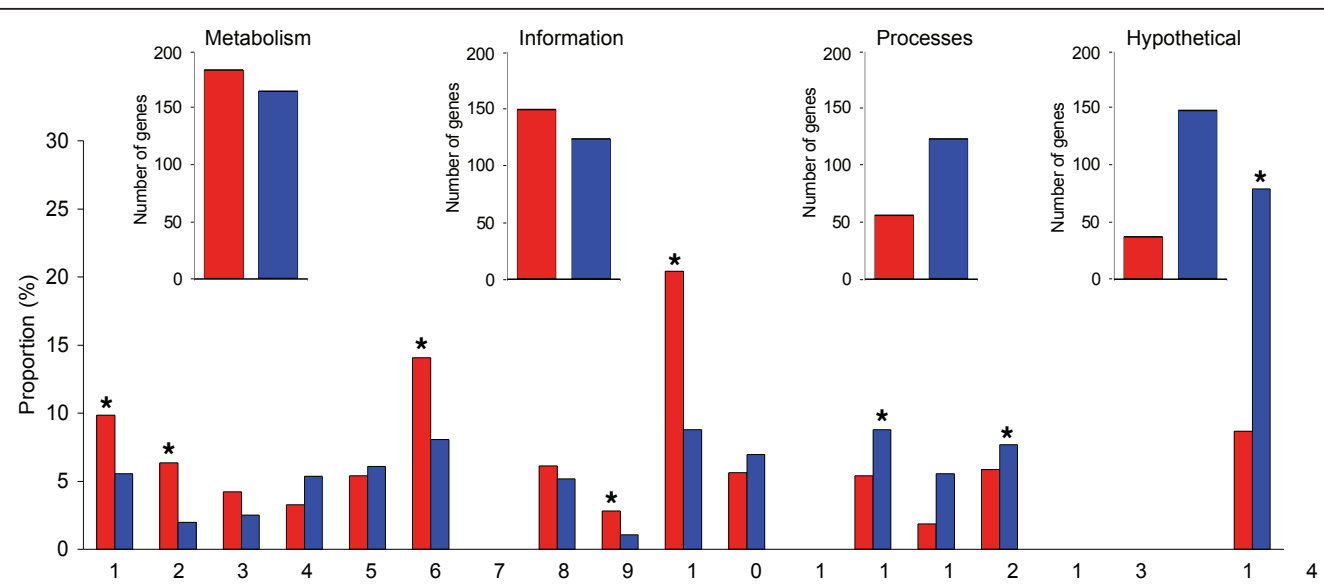

Figure 3 Functional distribution of orthologs divided by low or high nonsynonymous substitution rate (dN). The graph was generated using 985 orthologs with $\mathrm{dS}<5$; they were divided as described in the Methods section. Functions were grouped into Metabolism (1, amino acid biosynthesis; 2, nucleotide biosynthesis; 3, fatty acid biosynthesis; 4, cofactor biosynthesis; 5, central intermediary metabolism; and 6, energy generation), Information (7, DNA metabolism; 8, transcription; 9, translation; and 10, transcriptional regulators), Processes (11, transport; 12, cellular envelope synthesis; and 13, other cellular processes), and 14, Hypothetical functions. Bar colors: red, orthologs with low dN; blue, orthologs with high $\mathrm{dN}$. Asterisks denote significant differences with $p<0.05$, Fisher's exact test.

remaining possible tree combinations accounted for $11 \%$ of orthologs. A phylogenetic marker, such as $16 \mathrm{~S}$ rRNA supports the most abundant phylogeny RA-S-BM, in agreement with a closer association of $R$. etli and $A$. tumefaciens (Additional file 1) [26]. Other markers, such as AtpD (RS-A-BM), RecA (RA-S-BM) and DnaJ (RA-S$\mathrm{BM})$ were also found distributed in the main phylogenies.

\section{Physico-chemical properties of the ortholog products}

We applied the five series of values reported by Atchley et al. [9], to determine how the sequence variability of the orthologs affects the biochemical characteristics of the encoded proteins. Each series of values analyzes a special feature of the residues, namely polarity, secondary structure, volume, amino acid composition, and electrostatic charge [9]. The calculated values of the ortholog products spanned a wide range, from negative to positive values (with the exception of the amino acid composition, which only gave positive values). The electrostatic charge and volume showed the best correlation values (Pearson coefficient $\mathrm{r}=0.848$ in S. meliloti), followed by volume and amino acido composition $(\mathrm{r}=$ -0.662). We detected an atypical group of 110 protein sequences in polarity (for example, in the comparison with amino acid composition), consisting almost entirely of proteins with a high number of transmembrane segments (data not shown). The low polarity of membrane proteins has long been known [27], confirming the appropriate application of the method. The rest of the organisms showed similar tendencies (not shown).

With a clustering method [23], we tried to find relationships among species derived from the physico- chemical nature of the residues encoded by the orthologs. We generated clustering profiles for each property (shown for polarity, Figure 4A, and the rest in the Additional file 2) with the most conserved gene set (705 syntenic orthologs with $\mathrm{dS}<5$ ). A high level of variation among each ortholog can be observed, reflecting specific changes in each of the species. For polarity, a closer association of $R$. etli with $S$. meliloti, followed by $A$. tumefaciens in another branch, was observed (an RS-A$\mathrm{BM}$ association, Table 1); this relationship was similar to the second most abundant phylogeny described above. For the rest of the properties, other associations were observed: RA-S-BM for secondary structure, electrostatic charge, and volume (Additional file 2 and Table 1), and again RS-A-BM for amino acid composition (Table 1). These associations revealed which physico-chemical character links most closely with the species, reflecting the phylogenetic relationship and also the lifestyle of the organisms. When the complete 1416ortholog set was analyzed, B. melitensis departed clearly from the rest of species in polarity, charge and amino acid composition (Table 1, denoted as M-B in independent branches). In the group of Rhizobiales analyzed, Brucella is the only non free-living organism.

For a more detailed study of niche association by physico-chemical properties, we obtained the clustering for 17 alpha-proteobacterial species and 23 proteins, mainly from metabolism. The species group covered plant-associated organisms (Methylobacterium, Azospirillum), plant symbionts (the rhizobia), animal (Bartonella, Anaplasma) and plant (Agrobacterium) pathogens, and freeliving species (Caulobacter, Xanthobacter, Oligotropha, among others). The most clear cluster associations were 


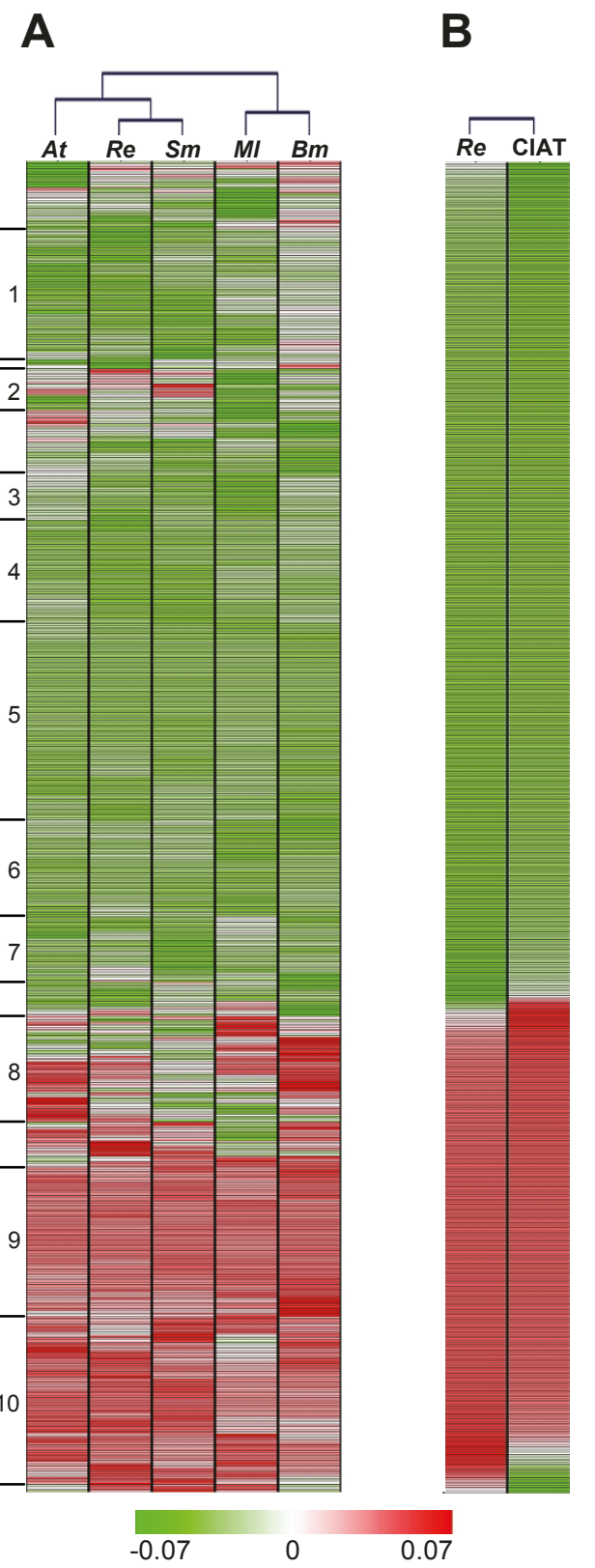

Figure 4 Association among species by physico-chemical characteristics of ortholog products. A, polarity is shown as example. The panel shows 705 syntenic ortholog products per species. Letters at the top: At, A. tumefaciens; Re, R. etli; Sm, S. meliloti; Bm, B. melitensis; and MI, M. loti. Lines at left denote clusters of orthologs with similar profile in the five species (ten were selected and analyzed in the text). B, comparison of two R. etli strains with polarity. The panel shows 705 syntenic ortholog products per strain. Letters at the top: Re, R. etli strain CFN42; CIAT, R. etli strain CIAT652. Clustering was performed with MeV TM4 [23]. Scales for normalized values (green for negative values, white for zero, and red for positive). for amino acid composition (with clusters containing only symbionts, free-living species, or pathogens), volume and electrostatic charge (with clearly defined clusters for symbionts or pathogens) (Additional file 3). In contrast, polarity and secondary structure showed a majority of phylogenetic associations.

To search for the relationships of physico-chemical associations with phylogeny, we determined the associations of each group of orthologs with a given phylogeny. We analyzed the most conserved group, 705 syntenic orthologs. In the case of 380 orthologs with phylogeny RA-S-BM, the same association was obtained for secondary structure, volume, and charge (Table 1). The orthologs with phylogeny RS-A-BM produced the same association for secondary structure, polarity, and amino acid composition. The orthologs with the phylogeny ASR-BM showed the same association for secondary structure and charge (Table 1). These data showed that secondary structure was the physico-chemical property most consistent with phylogeny (because each phylogenetic group showed the same association in that property); in contrast, for polarity, all groups reflected the same association (RS-A-BM), indicating a strong coherence of that property. For the rest of properties, the main phylogenetic groups (by number of orthologs, RA$\mathrm{S}-\mathrm{BM}$ or RS-A-BM) dominated the species association.

We analyzed in more detail groups of products from the polarity clustering (Figure 4A). Ten clusters were selected, comprising $83 \%$ of the total orthologs under analysis. Clusters 1, 4, 5, and 7 presented abundance in metabolic functions, while clusters 9 and 10 were abundant in informational functions. In concordance to their profile homogenity (red or green in Figure 4, positive or negative signs in the table of the Additional file 4), their members showed reduced levels of the percentage of changes by species (\%chSp) (Additional file 4). A cluster with more diverse levels, such as 8 , contained proteins pertaining to hypothetical functions, with high values of \%chSp (Additional file 4).

In order to have an assessment at the strain level, we compared the chromosomal orthologs of $R$. etli CFN42 with $R$. etli CIAT652, which shows better symbiotic performance than CFN42 $[18,28]$. We found an almost identical pattern of polarity between the two strains, but also found small groups of products with diverse values (Figure 4B), with a significant abundance of products belonging to the functional classes of cell wall synthesis, DNA metabolism and cellular processes (especially motility) (not shown).

Given the observed functional tendencies described above, we divided the products having the same sign in 
Table 1 Relationship of phylogeny and species association by physico-chemical property.

\begin{tabular}{lcccccc}
\hline & \multicolumn{5}{c}{ Association by physico-chemical property } \\
\hline Ortholog classes & Number of genes & Polarity & Secondary structure & Volume & Amino acid composition & Electrostatic charge \\
\hline Orthologs & 1416 & RS-A-M-B & RS-A-BM & RA-S-BM & RS-A-M-B & RA-S-M-B \\
Syntenic orthologs & 705 & RS-A-BM & RA-S-BM & RA-S-BM & RS-A-BM & RA-S-BM \\
\hline Syntenic orthologs divided by phylogeny & & & & & \\
RA-S-BM & 380 & RS-A-BM & RA-S-BM & RA-S-BM & RS-A-BM & RA-S-BM \\
RS-A-BM & 180 & RS-A-BM & RS-A-BM & RA-S-BM & RS-A-BM & RA-S-BM \\
AS-R-BM & 92 & RS-A-BM & AS-R-BM & RS-A-BM & RA-S-BM & AS-R-BM \\
\hline
\end{tabular}

Syntenic orthologs with phylogenies other than the main three shown (accounting for 53 products) were discarded from the analysis. Letters denote species: R: R. etli, S: S. meliloti, A: A. tumefaciens, B: Brucella melitensis, M: Mesorhizobium loti (a pair of letters denote that these species are in the same branch). Species association remarked in bold denotes equivalence with phylogeny.

the five species and obtained functional distributions. We found an interesting relationship between the functional role of the protein and its sign from the physicochemical property evaluation, which could be related to specific biochemical requirements for functional performance. For polarity, functions of metabolism and processes were related to negative signs, while informational functions were strongly related to positive signs (significant differences with $\mathrm{p}<0.05$, calculated with Fisher's exact test) (Figure 5). Several other remarkable features were found: for secondary structure, the negative sign (related to abundant alpha-helical structures) was associated with informational functions, and the positive sign (abundant coils) was related with synthesis of the cellular envelope and hypothetical proteins (Additional file 5). Hypothetical proteins showed significant abundance of low level amino acid composition (i. e., atypical composition); translation was abundant in protein sequences with positive charge; and energy and translation functional classes showed residues with high molecular volume (Additional file 5).

To find a relationship between the physico-chemical characteristics of proteins and evolutionary rates, we plotted distribution curves for gene groups with low or high $\mathrm{dN}$ and their physico-chemical values, for each of the properties. A striking general tendency was observed, because low $\mathrm{dN}$ genes presented significant abundance of sequences with positive sign in polarity, volume, and electrostatic charge (Additional file 6, significant differences in distribution curves, Mann-Whitney test, with $\mathrm{p}<0.05)$, revealing a specific physicochemical evolution of products.

To determine the relationship of the \%chSp with physico-chemical properties, we generated a dispersion graph, considering the average of the \%chSp and the range of polarity, as shown for example in Figure 6A. A positive relationship was obtained, revealing that sequence variability corresponded with diversity in the character evaluated (a similar tendency was observed in the rest of the properties, not shown). The dispersion was analyzed by obtaining clusters with high correlation values (see Methods). Strikingly, clear tendencies in molecular weight and isoelectric point were obtained. The molecular weight was progressively reduced according with a negative slope (Figure $6 \mathrm{~B}$ ), while the opposite was observed for isoelectric point (Figure 6C). Similar

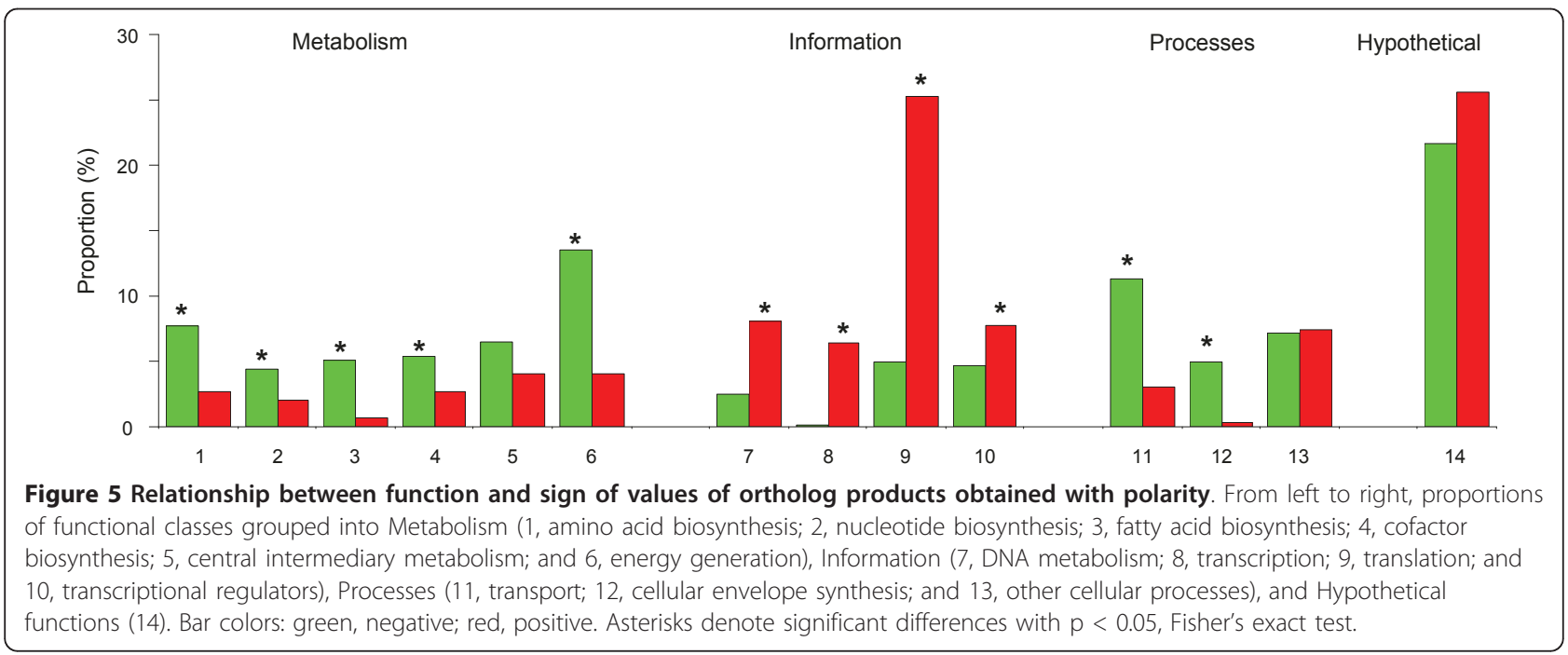



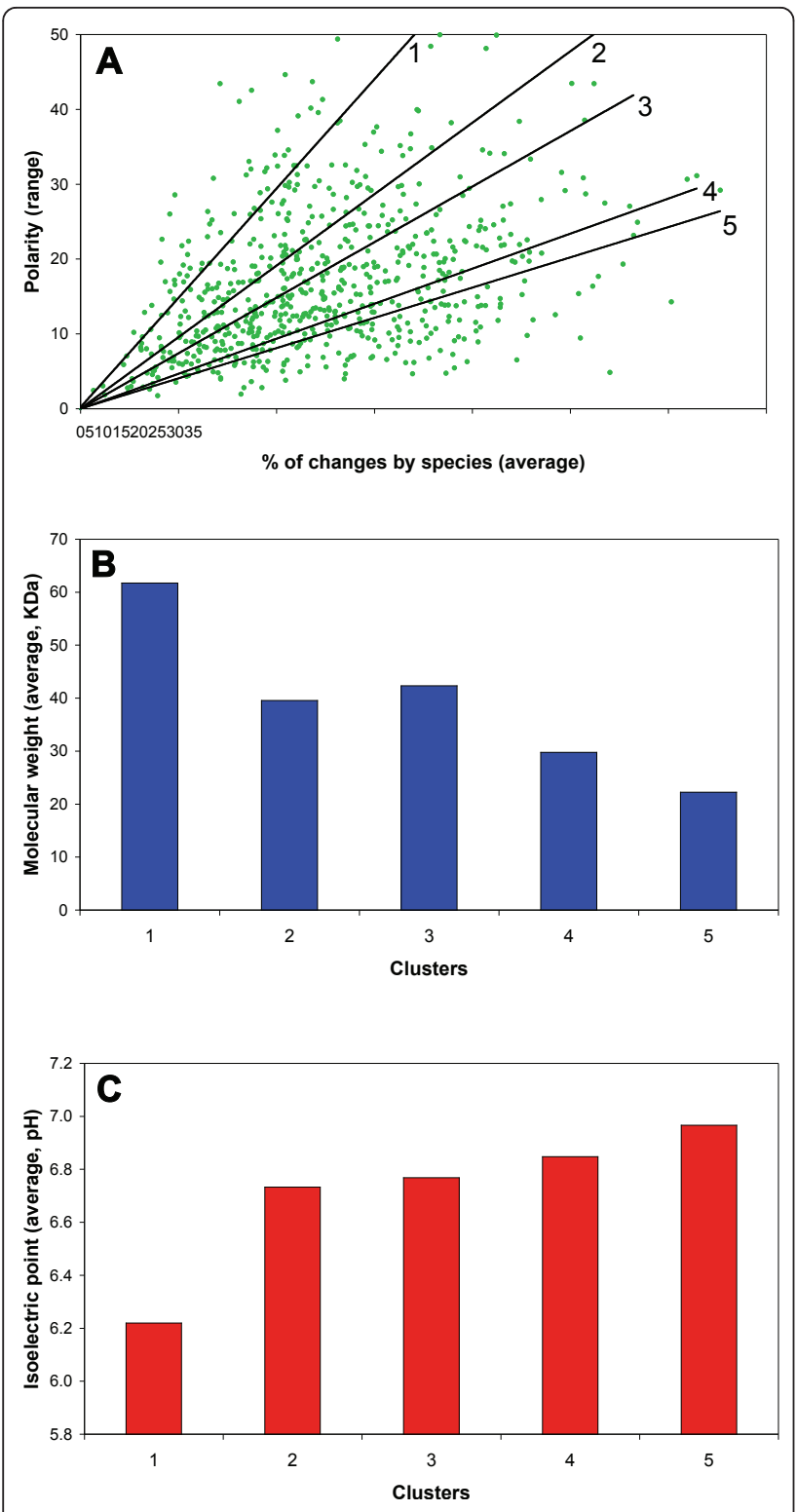

Figure 6 Relationship between the physico-chemical properties and the percentage of changes by species (\%chSp). A, dot plot graph of the average \%chSp and polarity (range). Tendency lines for the five clusters are shown. B, molecular weight (average) of syntenic ortholog products, following the order of the slope of clusters (up to down). C, isoelectric point (average) of syntenic ortholog products, following the order of the slope of clusters.

tendencies were observed in the three other properties, with the exception of electrostatic charge (not shown). The first cluster had significant abundance of proteins from the functional classes of amino acid biosynthesis, transcription and energy generation (Fisher exact test, $\mathrm{p}$ $<0.05$ ), and the last one was abundant with proteins of hypothetical functions and some cellular processes (not shown).
Lastly, we analyzed the data for a specific syntenic ortholog, $\arg C$, the gene that we used previously to experimentally validate the \%chSp approach. We reported the experimental effect of ortholog replacement in an S. meliloti $\operatorname{argC}$ mutant [29]. The $\operatorname{argC}$ from $M$. loti showed almost a complete inability to functionally complement the native gene in minimal medium supplemented with succinate-ammonium or mannose and nitrate [29]. The M. loti product showed the most differences in volume, secondary structure, and electrostatic charge, in comparison with S. meliloti (in the table of the Additional file 7). Possibly these three physico-chemical properties were important to define the phenotypic behavior of $M$. loti ArgC complementation. $R$. etli and A. tumefaciens genes, with similar divergence to $S$. meliloti, showed a better complementation phenotype but, interestingly, they also showed some differences. The A. tumefaciens gene produced a reduced growth ability and exhibited a strong excretion of metabolites, resembling the $\operatorname{argC}$ mutant strain [29]. The $A$. tumefaciens product was more similar to that of $M$. loti in charge and volume (Additional file 7). On the other hand, the $R$. etli gene showed a growth phenotype most resembling that of $S$. meliloti, and the products from $R$. etli and S. meliloti had identical values in volume, and some similar values in charge; therefore, these properties can be considered as the most important physico-chemical factors in determining the observed complementation efficiency.

\section{Discussion}

Several studies have focused on essential gene recognition and evaluation of evolutionary rates [30-36], but few studies in bacteria have analyzed the relationship of evolutionary rates and phylogeny with the functional role and the physico-chemical properties of a massive group of proteins. We used new parameters to evaluate these traits, including the percentage of changes by species (\%chSp), and a series of values for physico-chemical characteristics of amino acid residues; also, we consider our group of orthologs (mainly syntenic genes), which perform housekeeping functions and also a diversity of cellular tasks, as an adequate gene set to study the conserved characteristics among related species, but also to analyze the ortholog variability and relationship with function and niche adaptation.

We analyzed 7080 genes (organized in 1416 sets of five values each) evaluating how their sequence variability could affect the physico-chemical characteristics of the encoded proteins. Previously, we proposed a parameter, the \%chSp, to extract species-specific information that may be related to the cellular milieu and niche adaptation [4]. We showed the values and distribution 
of the \%chSp of the chromosomal orthologs (two groups of species were formed), and how the functional classes showed differential amounts of \%chSp (Figure 1). Strikingly, we found a very high correlation of this parameter with codon evolution (Figure 2), mainly nonsynonymous substitution rate $(\mathrm{dN})$, indicating that the \%chSp is an efficient way to obtain important evolutive information directly from the protein sequence. Some homologous positions in multiple alignments of proteins were reported earlier to be associated with functional variation [37].

Most importantly, non-neutral variations in genes can change the potential of the cell for further adaptations [5,38-41], and even neutral modifications, as explained in the frame of the constructive neutral evolution, about fixation of neutral or slightly deleterious mutations [42]. In our case, protein sequences of orthologs presented different values in each of the five physico-chemical series of values (Figure 5 with polarity as example, and more pronounced in the rest of properties, Additional file 5), that reflected the vast amount of specific changes in each ortholog and species, possibly responding again to the intracellular milieu and niche adaptation. In the comparison of orthologs belonging to $R$. etli strains $[18,28]$, very few changes were observed (polarity as an example, Figure 4B), demonstrating the overall closeness of these organisms. We propose that variability of orthologs determine key properties of the proteins. We found that changes in orthologs indeed define particular physico-chemical characteristics. In this sense, these findings indicate that changes in orthologs (mainly nonsynonymous substitutions), defined commonly as neutral [5,43], determine particular physico-chemical characteristics that lead to alterations and represent functional requirements. In this context, it was found that most of the mutations in ribosomal protein genes were similar and weakly deleterious but, mostly important, the distribution of fitness for synonymous and nonsynonymous mutations were similar [44].

In an experimental approach in our laboratory, we substituted an essential syntenic ortholog $(\arg C)$ from the arginine biosynthetic pathway of Sinorhizobium meliloti, and expressed $\operatorname{argC}$ orthologs from other Rhizobiales in that background, with the aim of demonstrating the relationships of particular residues with function and evolution. By a physiological, enzymatic, transcriptional, and proteomic characterization we found that several factors, such as the type of promoter and the percentage of changes by species, determined different functionality and levels of transcription and translation of the ArgC protein [29]. We used the physico-chemical series of values obtained by Atchley, to correlate the physico-chemical properties of the proteins with the real phenotype and performance of
$\operatorname{argC}$ orthologs, in a $S$. meliloti background. As observed in Additional file 7, the R. etli ortholog, with more similar physico-chemical values to $S$. meliloti, showed the most resembling phenotype; followed by the $A$. tumefaciens gene, the $M$. loti $\arg C$ showed a severe growth deficiency and very different physicochemical values, in comparison with $S$. meliloti. As Dr. Koonin and collaborators said regarding our work [45], it is necessary to consider that functional conservation among orthologs should be inferred with caution because some orthologs genes can diverge functionally even among closely related organisms. Also, there are other factors that influence adaptation in bacteria, such as compensatory gene amplification after orthologous replacement [46], which was also found in our work.

Most essential functions, such as information and metabolism were significantly covered by low $\mathrm{dN}$ genes in the five species, showing more restriction to evolution; in contrast, orthologs with high $\mathrm{dN}$ were functionally more flexible, abundant in hypothetical functions and cellular processes, but perhaps also showed more dispensability (Figure 3). The differences in evolutionary rates of genes have been adscribed to functional constraints, but a debate is open to clarify the importance of translational costs, reflected in misfolding [47], structural factors $[48,49]$, and even pleiotropy [50], as the factors influencing the evolution of proteins. The compositional and adaptation codon bias in genes are important characteristics of GC-rich organisms, such as the Rhizobiales, because these traits are related to increased expressivity [51-53]. We found that low $\mathrm{dN}$ genes had high CAI values (not shown) showing, as others have, that codon bias is a selective force in these genes and a negative correlation of expressivity and evolutionary rates exists [54-56].

We found that the series of values reported by Atchley [9] were very useful to discern the contribution of physico-chemical factors of amino acids to the characteristics of the ortholog products. These values summarize the amino acid features into five biochemical characteristics, namely polarity, secondary structure, amino acid composition, molecular volume, and electrostatic charge [9]. We found the global correlations among the physico-chemical properties that can be possibly generalized to all bacterial proteins. Interestingly, we found a group of products, clearly differentiated from the rest by polarity, composed almost exclusively by proteins with many transmembranal segments (not shown). This result confirmed that these series of values can detect specific features of residues forming part of proteins. Recently, Marsella et al. [57], using the series of values reported by Atchley detected an especial periodicity of solenoid secondary structures. In another study, the same series 
of values were used to define niche adaptation of prokaryotic organisms from extreme environments [58].

When orthologs were related by their physico-chemical features, species associations were similar to the most abundant phylogenies, indicating a link between characteristics of proteins and species evolution (Figure 4A, and Additional file 2); however, they also showed differences that may account for other structural factors related to intra- and extra-cellular environments in shared niches, for example $R$. etli products most closely related to those from $S$. meliloti in polarity and amino acid composition (possibly because both organisms are symbionts), but most closely to A. tumefaciens products in secondary structure, volume, and electrostatic charge (in this case, close phylogenetically relatedness can be argued). These relationships shed light about the importance of considering an approach such as that described here. In this regard, in comparison with classical phylogeny, the physico-chemical approach of species association can give an additional amount of information. $B$. melitensis orthologs, despite their closer phylogenetic relationship with $R$. etli, S. meliloti and A. tumefaciens, departed from the other four species in amino acid composition, charge and polarity (not shown), possibly reflecting differential adaptation in this set of orthologs; Brucella is the only species of the analyzed with a non free lifestyle. Recently, it was reported that B. melitensis has a more basic proteome than the rest of Rhizobiales, possibly in response to its intracellular lifestyle [59], in addition to being subjected to genomic erosion processes (such as gene loss, pseudogenization, and reduction of CAI), like other intracellular organisms [60-63]. In the clustering analysis covering many alpha-proteobacterial species and their typical lifestyles, clear niche associations were found for some properties (Additional file 3).

A remarkable result was the observation that orthologs with low nonsynonymous substitution rate $(\mathrm{dN})$ were associated with specific amino acid characters, such as higher polarity and electrostatic charge, and large molecular volume (Additional file 6), possibly reflecting physico-chemical tendencies of the evolution of genes. Additionally, the functions of orthologs were related with the particular sign of values from physicochemical property evaluation (Figure 5, and Additional file 5), indicating more or less strict specific biochemical requirements of the residues in the proteins to perform their role. In some cases, there were clear differences between the metabolic and informational functions, or between the metabolic and processes functions. Furthermore, some of the hypothetical protein sequences appeared with the same sign as that of metabolic genes or genes encoding proteins involved in processes; the qualification with these series of values would be useful in the eventual functional characterization of the hypothetical proteins. Additionally, interesting tendencies were found regarding molecular weight and isoelectric point, along with the increase of the percentage of changes by species (Figure 6). These findings clearly merit a more detailed analysis.

\section{Conclusions}

In this work, we found that the sequence variability of the chromosomal orthologs of the Rhizobiales species under study have evolutionary and functional implications, closely related to the physico-chemical properties of the products. We found that the percentage of changes by species of the orthologs directly reflects the nonsynonymous substitution rate; and that there was a relationship of the physico-chemical characteristics of residues encoded in the proteins with evolution and specific functional requirements. This genomic study contributes to the understanding of factors that connect the evolution of genes with the physico-chemical characteristics of the proteins and their functional adaptation.

\section{Reviewers comments}

Reviewer report 1. Dr. Purificación López-García, Centre National de la Recherche Scientifique, France

Peralta and co-workers present a comparative analysis of 1416 orthologous proteins shared by five species of Rhizobiales, three plant symbionts, one plant pathogen and one animal pathogen. After carrying out phylogenetic analyses of those orthologs and finding three major topologies, Peralta et al. study the sequence variability of a subset of these proteins by looking at the number of synonymous $(\mathrm{dS})$ and non-synonymous $(\mathrm{dN})$ substitutions and a related measurement, the "species signature" defined as the number of species-specific amino acid residues. The authors study possible correlations between protein sequence variability and a series of associated properties (polarity, electrostatic charge, secondary structure, molecular volume and amino acid composition) and find particular trends, which suggest that particular physico-chemical properties are associated with particular functional roles. The authors further speculate that much sequence variation is not related to phylogeny but to adaptation to particular niches. Although the last conclusion needs further testing, the overall work is properly done and interesting and I have only some relatively minor comments.

Author's response: Thanks for your commentary. Effectively, the question about how much of the divergence belong to neutral variation and what amount to adaptation is our departure point for the analysis. For a long time, we have wondered about how to disect the problem. We consider that our approach is useful to analyze in detail the sequence variability and we are beginning 
to find answers about this issue. Please, also see our response for the last question.

My first comment relates to the "species signature". The authors find "striking" to see a "correlation of this parameter with codon evolution, mainly nonsynonymous substitution rate $(\mathrm{dN})$, indicating that the species signature is efficient to obtain important evolutive information, directly from the protein sequence". This is not striking at all since, even if $\mathrm{dN}$ is estimated from DNA sequence, it corresponds in fine to changes in amino acid sequence, so that the "species signature" is but a subset of the amino acid changes resulting from nonsynonymous substitutions $(\mathrm{dN})$. It is normal that they both contain evolutionary information because the two measures are correlated. What I do not understand well is the advantage of the "species signature" versus $\mathrm{dN}$. The number of non-synonymous substitutions is independent of taxonomic sampling, but the "species signature" depends on taxonomic sampling. By contrast to $\mathrm{dN}$, as more and more strains or species genome sequences become available within a genus, the "species signature" will most likely progressively decrease and eventually reach zero (especially if one considers orthologs for a same protein from the whole tree of life). In this sense, the use of the "species signature" seems limited and restricted to particular case studies.

Author's response: We eliminated the word "striking" in the beginning of the mentioned paragraph. Our proposal of species signature is devoted to distinguish certain differences in closely related genomes. The signature reaches zero when sequences from organisms show less phylogenetic differences; $i$. e., ideally when they form part of the same species.

The authors show that changes in orthologs correlate with particular physico-chemical properties of the corresponding proteins and, hence, likely functional adaptation to particular niches. However, this correlation remains at a theoretical, predictive level. Since the studied proteins are annotated and their functions known, it would be very illustrative if the authors could point at one or several particular examples and explain them in the context of their analysis.

Author's response: With the last analysis in the Results section, we tried to relate the phenotypic experimental differences of the argC complementing strains with its physico-chemical properties.

As stated in the Discussion section, even when each ortholog performs the same function in a given genome, without doubt the gene (and its product) must be adapted in order to respond to a particular physiological environment.

Although the mentioned proposal seems speculative, it is important to take into account our recent publication
(Diaz et al., J. Bacteriol. 193:460-472, 2011), in which our results from complementation experiments showed that the substitution of the S. meliloti argC gene, with the corresponding ortholog from other Rhizobiales species, was not completely functional, and also affected the growth, transcription rate and translation, and possibly the whole cellular physiology. Indeed, as Dr. Koonin said recently of our work (Kristensen, et al., Brief. Bioinf., 2011, doi:10/1093/bib/bbr030), it is necessary to consider that functional conservation among orthologs should be inferred with caution, because some orthologous genes can diverge functionally even among closely related organisms. As a matter of fact, this manuscript was the initial approach that allowed us to design the experiments of the already published work.

Reviewer report 2. Prof. Jeffrey Townsend (nominated by Prof. J. Peter Gogarten), Yale University, United States The revised manuscript clarifies the "species signature" metric used, and addresses issues related to its comparison to $\mathrm{dN}$ fairly satisfactorily. The manuscript does not improve or extend its results by applying either of the two approaches recommended that would considerably empower the conclusions being drawn.

From the original review:

First, the relationships between physico-chemical characteristics of residues encoded and functional category of the gene could be analyzed with more specific and powerful statistical approaches. In particular, instead of dividing a continuous range arbitrarily into two discrete categories to perform purely categorical statistical analyses, a more powerful approach would consist of performing logistic regressions with the continuous variable on the $\mathrm{x}$-axis and the categorical on the $\mathrm{y}$ (i.e. high or low $\mathrm{dN}$ as a continuous predictor of functional category). Second, the manuscript addresses gene-wide evolutionary rate and correlates it to gene-wide physicochemical metrics. Because each of these metrics is "averaged" across a gene, considerable power to detect important signal is lost. A more direct way to assess these questions would be to correlate evolutionary rates of individual sites and physico-chemical properties of those sites. Examining the rates of evolution of sites and of physico-chemical properties of amino acids could also better clarify the phylogenetic discrepancies among orthologs noted in this manuscript. These discrepancies among inferences from the orthologs are noted, but are attributed without much detail to selection based on ecological niche, and are not directly evaluated in terms of potential phylogenetic signal, noise, or horizontal gene transfer of the gene sequences, all of which may play roles in the different results achieved when analyzed solo for phylogenetic inference. 


\section{Reviewer report 3. Ms. Olga Kamneva, University of Wyoming, United States}

The authors analyzed the set of orthologous gene families from five strains of five different species of Rhizobiales using several techniques, the authors aim to characterize patterns of sequence evolution in relation to physical properties of encoded proteins within gene families and examine how change of protein physicochemical properties is related to niche adaptation.

I think that while the authors had an interesting and novel idea, to investigate how the physical properties of evolutionary related proteins are changed across related organisms of distinct life styles I feel that quality of the study can be significantly improved by shifting the focus of the study from trying to find correlation between change of physical properties and evolutionary rates to characterizing mechanisms and consequences of change of the physical properties. I also have some technical comments.

Specific comments:

1. The study should benefit from using one of the clustering based approaches to identify gene families with subsequent inference of orthologs. Using bidirectional best hit for this leads to under-determination of inparalogs within orthologous groups, as well as including xenologs into the gene families.

2. The study would also benefit from using modern phylogeny inference software (PhyML or RaxML), the results should not differ much but will be more robust.

3. A dS threshold value of 5 is too high, dS values larger than 3 is considered to be a sign of synonymous sites saturation even when estimated using maximum likelihood [Z. Yang, personal communication http://gsf. gc.ucdavis.edu/viewtopic.php?f=1\&t=2291\&p=3967\&hilit=saturation \&sid=224cf845cae40709bad44196fb33ffed\#] Also the threshold should be applied to the depth of the whole tree, not just certain branches. If synonymous sites are saturated dS will be determined wrong and it does not matter what it is used for, $\mathrm{dN} / \mathrm{dS}$ calculation or to see how well $\mathrm{dS}$ correlates with something else (not dN).

4. The authors use "percentage of changes by species" as measure of species-specific protein sequence variation. As it is explained in the manuscript and follows from the name of the metric, "percentage of changes by species" is a percentage of the protein alignment columns where the sequence from one particular species has a different residue than the rest of the alignment column. Basically "percentage of changes by species" is a crude approximation of the number of amino acid substitutions that occurred on a branch leading to the sequence over the total length of the sequence. The only benefit of the used metric is the ease of calculation, while terminal branch length of protein gene trees (or
$\mathrm{dN}$ trees) appears to be an estimate of the same thing and be technically more rigorous. I suggest not using "percentage of changes by species" for this study at all.

5 . Figure 2 in my opinion should be omitted as well as use of "percentage of changes by species".

6. I like the idea of clustering organisms based on the physical properties of proteins in gene families (Figure 5 and Additional file 3). Correlation between life style and proteome properties was shown before [Tekaia $\mathrm{F}$ et al. Gene. 2002.], ref [58,59] of the manuscript, while underlying biology of these observed patterns is unknown. Looking at changes of physical properties of evolutionary related proteins from organisms of different life style is novel. However with only one animal pathogen and one plant pathogen in the set (Figure 5), it is not possible to recover something meaningful while on the Additional file 3 only clustering based on amino acid composition is clear. Thus I am not sure if conclusion about correlation of protein properties and species ecology has much of support.

7. Figure 5 shows proteins which are extremely polar in one genome and extremely non-polar in another. It is interesting to see how this shift in polarity or any other property occurs. Is it caused by an additional domain which perturbs the average or it is due to point mutations? It is also interesting to see how this shift affects structure/function of the proteins. 1-2 case studies should be shown (alignments, comparison of protein structures, conservation/alteration of functionally important residues). It would be a first attempt to reveal molecular mechanisms underlying the correlation between ecology of microbes and properties of their proteomes.

\section{Additional material}

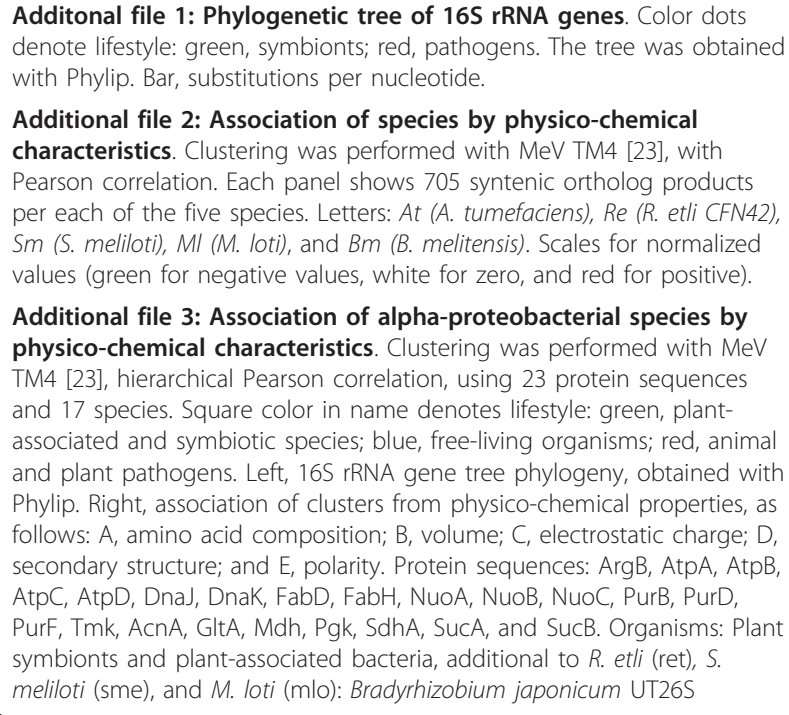


(designated as bja, GenBank accession no. NC_014013.1), Azorhizobium caulinodans ORS571 (azc, NC_009937.1), Methylobacterium extorquens AM1 (mea, NC_012808.1), Azospirillum sp. B510 (azl, NC_013854.1). Free living species: Xanthobacter autotrophicus Py2 (xau, NC_009720.1), Rhodopseudomonas palustris BisA53 (rpe, NC_008435.1), Oligotropha carboxidovorans OM5 (oca, NC_015684.1), Nitrobacter winogradsky Nb-255 (nwi, NC_007406.1), Caulobacter crescentus CB15 (ccr, NC_002696.2). Animal and plant pathogens, additional to A. tumefaciens (atu) and B. melitensis (bme): Ochrobactrum anthropi ATCC 49188 (oan, NC_009668.1), Bartonella henselae Houston-1 (bhe, NC_005956.1), and Anaplasma centrale Israel (acn, NC_013532.1). Bars denote substitutions per nucleotide (16S rRNA tree) and arbitrary distances (physico-chemical properties), respectively.

\section{Additional file 4: Characteristics of selected groups of products} from clustering of polarity

Additional file 5: Relationship between function and sign of values of ortholog products obtained with the series of values of physicochemical properties. In the top row (from left to right), number of orthologs grouped into Metabolism (1, amino acid biosynthesis; 2 , nucleotide biosynthesis; 3, fatty acid biosynthesis; 4 , cofactor biosynthesis; 5 , central intermediary metabolism; and 6 , energy generation), Information (7, DNA metabolism; 8, transcription; 9, translation; and 10, transcriptional regulators), Processes (11, transport; 12, cellular envelope synthesis; and 13, cellular processes), and Hypothetical functions (14). In the bottom row, the functional classes in proportions. Asterisks denote significant differences with $p<0.05$, Fisher's exact test. Bar colors: green, negative (low level for amino acid composition); red, positive (high level for amino acid composition). Scale for amino acid composition graph was broken to conserve the shape.

Additional file 6: Relationships between the nonsynonymous substitution rate $(\mathrm{dN})$ and the physico-chemical properties.

Frequency distribution curves of the orthologs divided in groups with low or high dN values. A, polarity. B, volume. C, electrostatic charge. Data from S. meliloti products. Low dN group, orange line. High dN group, green line.

Additional file 7: Values of physico-chemical properties for ArgC from Rhizobiales.

\section{Acknowledgements and funding}

We thank Julio Martínez for help with statistics, and Julio Collado, Michael Dunn and Yolanda Mora (CCG-UNAM) for reviewing the manuscript. This work was partially supported by the Dirección General de Asuntos del Personal Académico (DGAPA-Universidad Nacional Autónoma de México), grants IN215307 and IN212710.

\section{Authors' contributions}

$\mathrm{JM}$ and $\mathrm{HP}$ conceived of the project. HP, GG, AA, and JM performed the analysis and analyzed the results. HP and JM wrote the draft manuscript. All authors read and approved the final manuscript.

\section{Competing interests}

The authors declare that they have no competing interests.

Received: 11 April 2011 Accepted: 4 October 2011

Published: 4 October 2011

\section{References}

1. Hulsen T, Hynen MA, De Vlieg J, Groenen PMA: Benchmarking ortholog identification methods using functional genomics data. Genome Biol 2006, 7(4):R31

2. Huynen MA, Bork P: Measuring genome evolution. Proc Natl Acad Sci USA 1998, 95:5849-5856.

3. Zheng XH, Lu F, Wang ZY, Zhong F, Hoover J, Mural R: Using shared genomic synteny and shared protein functions to enhance the identification of orthologous gene pairs. Bioinformatics 2005, 21(6):703-710
4. Guerrero G, Peralta H, Aguilar A, Diaz R, Villalobos MA, Medrano-Soto A, Mora J: Evolutionary, structural and functional relationships revealed by comparative analysis of syntenic genes in Rhizobiales. BMC Evol Biol 2005, 5:55.

5. Soskine M, Tawfik DS: Mutational effects and the evolution of new protein functions. Nat Rev Genet 2010, 11:572-582.

6. Hughes AL, Nei M: Pattern of nucleotide substitution at major histocompatibility complex class I loci reveals overdominant selection. Nature 1988, 335:167-170.

7. Yang Z, Bielawski JP: Statistical methods for detecting molecular adaptation. Trends Ecol Evol 2000, 15:496-503.

8. Yang Z: Maximum-likelihood models for combined analyses of multiple sequence data. J Mol Evol 1996, 42:587-596.

9. Atchley WR, Zhao J, Fernandes AD, Drüke T: Solving the protein sequence metric problem. Proc Natl Acad Sci USA 2005, 102(18):6395-6400.

10. Wood DW, Setubal JC, Kaul R, Monks DE, Kitajima JP, Okura VK, Zhou Y, Chen L, Wood GE, Almeida NF Jr, Woo L, Chen Y, Paulsen IT, Eisen JA, Karp PD, Bovee D Sr, Chapman P, Clendenning J, Deatherage G, Gillet W, Grant C, Kutyavin T, Levy R, Li MJ, McClelland E, Palmieri A, Raymond C, Rouse G, Saenphimmachak C, Wu Z, Romero P, Gordon D, Zhang S, Yoo H, Tao Y, Biddle P, Jung M, Krespan W, Perry M, Gordon-Kamm B, Liao L, Kim S, Hendrick C, Zhao ZY, Dolan M, Chumley F, Tingey SV, Tomb JF, Gordon MP, Olson MV, Nester EW: The genome of the natural genetic engineer Agrobacterium tumefaciens C58. Science 2001, 294(5550):2317-2323.

11. DelVecchio VG, Kapatral V, Redkar RJ, Patra G, Mujer C, Los T, Ivanova N, Anderson I, Bhattacharyya A, Lykidis A, Reznik G, Jablonski L, Larsen N, D'Souza M, Bernal A, Mazur M, Goltsman E, Selkov E, Elzer PH, Hagius S, O'Callaghan D, Letesson JJ, Haselkorn R, Kyrpides N, Overbeek R: The genome sequence of the facultative intracellular pathogen Brucella melitensis. Proc Natl Acad Sci USA 2002, 99(1):443-448.

12. Goodner B, Hinkle G, Gattung S, Miller N, Blanchard M, Qurollo B, Goldman BS, Cao Y, Askenazi M, Halling C, Mullin L, Houmiel K, Gordon J, Vaudin M, lartchouk O, Epp A, Liu F, Wollam C, Allinger M, Doughty D, Scott C, Lappas C, Markelz B, Flanagan C, Crowell C, Gurson J, Lomo C, Sear C, Strub G, Cielo C, Slater S: Genome sequence of the plant pathogen and biotechnology agent Agrobacterium tumefaciens C58. Science 2001, 294(5550):2323-2328.

13. Paulsen IT, Seshadri R, Nelson KE, Eisen JA, Heidelberg JF, Read TD, Dodson RJ, Umayam L, Brinkac LM, Beanan MJ, Daugherty SC, Deboy RT, Durkin AS, Kolonay JF, Madupu R, Nelson WC, Ayodeji B, Kraul M, Shetty J, Malek J, Van Aken SE, Riedmuller S, Tettelin H, Gill SR, White O, Salzberg SL, Hoover DL, Lindler LE, Halling SM, Boyle SM, Fraser CM: The Brucella suis genome reveals fundamental similarities between animal and plant pathogens and symbionts. Proc Natl Acad Sci USA 2002, 99(20):13148-13153.

14. Halling SM, Peterson-Burch BD, Bricker BJ, Zuerner RL, Qing Z, Li LL, Kapur V, Alt DP, Olsen SC: Completion of the genome sequence of Brucella abortus and comparison to the highly similar genomes of Brucella melitensis and Brucella suis. J Bacteriol 2005, 187(8):2715-2726.

15. Alsmark CM, Frank AC, Karlberg EO, Legault BA, Ardell DH, Canbäck B, Eriksson AS, Näslund AK, Handley SA, Huvet M, La Scola B, Holmberg M, Andersson SG: The louse-borne human pathogen Bartonella quintana is a genomic derivative of the zoonotic agent Bartonella henselae. Proc Natl Acad Sci USA 2004, 101(26):9716-9721.

16. Galibert F, Finan TM, Long SR, Puhler A, Abola P, Ampe F, Barloy-Hubler F, Barnett MJ, Becker A, Boistard P, Bothe G, Boutry M, Bowser L, Buhrmester J, Cadieu E, Capela D, Chain P, Cowie A, Davis RW, Dreano S, Federspiel NA, Fisher RF, Gloux S, Godrie T, Goffeau A, Golding B, Gouzy J, Gurjal M, Hernandez-Lucas I, Hong A, Huizar L, Hyman RW, Jones T, Kahn D, Kahn ML, Kalman S, Keating DH, Kiss E, Komp C, Lelaure V, Masuy D, Palm C, Peck MC, Pohl TM, Portetelle D, Purnelle B, Ramsperger U, Surzycki R, Thebault P, Vandenbol M, Vorholter FJ, Weidner S, Wells DH, Wong K, Yeh KC, Batut J: The composite genome of the legume symbiont Sinorhizobium meliloti. Science 2001, 293(5530):668-672.

17. Kaneko T, Nakamura Y, Sato S, Asamizu E, Kato T, Sasamoto S, Watanabe A, Idesawa K, Ishikawa A, Kawashima K, Kimura T, Kishida Y, Kiyokawa C, Kohara M, Matsumoto M, Matsuno A, Mochizuki Y, Nakayama S, Nakazaki N, Shimpo S, Sugimoto M, Takeuchi C, Yamada M, Tabata S: Complete genome structure of the nitrogen-fixing symbiotic bacterium Mesorhizobium loti. DNA Res 2000, 7(6):331-338. 
18. González V, Santamaría RI, Bustos P, Hernández-González I, Medrano-Soto A, Moreno-Hagelsieb G, Janga SC, Ramírez MA, Jiménez-Jacinto V, ColladoVides J, Dávila G: The partitioned Rhizobium etli genome: genetic and metabolic redundancy in seven interacting replicons. Proc Natl Acad Sci USA 2006, 103(10):3834-3839.

19. Pearson WR, Lipman DJ: Improved tools for biological sequence comparison. Proc Natl Acad Sci USA 1988, 85(8):2444-2448.

20. Thompson JD, Higgins DG, Gibson TJ: CLUSTALW: improving the sensitivity of progressive multiple sequence alignment through sequence weighting, position-specific gap penalties and weight matrix choice. Nucl Acids Res 1994, 22(22):4673-4680.

21. PHYLIP Program for inferring phylogenies. [http://evolution.genetics. washington.edu/phylip.html].

22. Yang Z: PAML: a program package for phylogenetic analysis by maximum likelihood. Comput Appl Biosci 1997, 13(5):555-556.

23. Saeed Al, Sharov V, White J, Li J, Liang W, Bhagabati N, Braisted J, Klapa M, Currier T, Thiagarajan M, Sturn A, Snuffin M, Rezantsev A, Popov D, Ryltsov A, Kostukovich E, Borisovsky I, Liu Z, Vinsavich A, Trush V, Quackenbush J: TM4: a free, open-source system for microarray data management and analysis. Biotechniques 2003, 34(2):374-378.

24. Olivares-Hernandez R, Bordel S, Nielsen J: Codon usage variability determines the correlation between proteome and transcriptome fold changes. BMC Syst Biol 2011, 5:33

25. Persson $B$, Argos P: Prediction of transmembrane segments in proteins utilising multiple sequence alignments. J Mol Biol 1994, 237(2):182-192.

26. Llóret L, Martínez-Romero E: Evolucion y filogenia de Rhizobium. Rev Lat Microbiol 2005, 47:43-60.

27. Capaldi RA, Vanderkooi G: The low polarity of many membrane proteins Proc Natl Acad Sci USA 1972, 69(4):930-932.

28. González V, Acosta JL, Santamaría Rl, Bustos P, Fernández JL, HernándezGonzález IL, Díaz R, Flores M, Palacios R, Mora J, Dávila G: Conserved symbiotic plasmid DNA sequences in the multireplicon pangenomic structure of Rhizobium etli. Appl Environ Microbiol 2010, 76(5):1604-1614.

29. Díaz R, Vargas-Lagunas C, Villalobos MA, Peralta H, Mora Y, Encarnación S, Girard L, Mora J: $\arg C$ orthologs from Rhizobiales show diverse transcriptional efficiency and functionality in Sinorhizobium meliloti. $J$ Bacteriol 2011, 193:460-472.

30. Jordan IK, Rogozin IB, Wolf YI, Koonin EV: Microevolutionary genomics of bacteria. Theor Popul Biol 2002, 61(4):435-447.

31. Jordan IK, Rogozin IB, Wolf YI, Koonin EV: Essential genes are more evolutionarily conserved than are nonessential genes in bacteria. Genome Res 2002, 12(6):962-968.

32. Jordan IK, Wolf $\mathrm{Yl}$, Koonin EV: No simple dependence between protein evolution rate and the number of protein-protein interactions: only the most prolific interactors tend to evolve slowly. BMC Evol Biol 2003, 3:1.

33. Koonin EV: Comparative genomics, minimal gene sets, and the last universal common ancestor. Nat Rev Microbiol 2003, 1:127-136.

34. Danchin A: Genomes and evolution. Curr Issues Mol Biol 2003, 5:37-42.

35. Young JP, Crossman LC, Johnston AW, Thomson NR, Ghazoui ZF, Hull KH, Wexler M, Curson AR, Todd JD, Poole PS, Mauchline TH, East AK, Quail MA, Churcher C, Arrowsmith C, Cherevach I, Chillingworth T, Clarke K, Cronin A, Davis P, Fraser A, Hance Z, Hauser $H$, Jagels K, Moule S, Mungall K, Norbertczak H, Rabbinowitsch E, Sanders M, Simmonds M, Whitehead S, Parkhill J: The genome of Rhizobium leguminosarum has recognizable core and accessory components. Genome Biol 2006, 7(4):R34.

36. Koonin EV, Wolf Yl: Genomics of bacteria and archaea: the emerging dynamic view of the prokaryotic world. Nucl Acids Res 2008, 36:6688-6719.

37. Philippe H, Casane D, Gribaldo S, Lopez P, Meunier J: Heterotachy and functional shift in protein evolution. IUBMB Life 2003, 55:257-265.

38. Amitai G, Gupta RD, Tawfik DS: Latent evolutionary potentials under the neutral mutational drift of an enzyme. HFSP J 2007, 1:67-78.

39. Wagner A: Neutralism and selectionism: a network-based reconciliation. Nat Rev Gen 2008, 9:965-974.

40. Wagner A: Robustness, evolvability, and neutrality. FEBS Letters 2005, 579:1772-1778.

41. Chamary JV, Parmley JL, Hurst LD: Hearing silence: non-neutral evolution at silent sites in mammals. Nat Rev Genet 2006, 7:98-108.

42. Gray MW, Lukes J, Archibald JM, Keeling PJ, Doolittle WF: Irremediable complexity? Science 2010, 330:920-921.
43. Ortlund EA, Bridgham JT, Redinbo MR, Thornton JW: Crystal structure of an ancient protein: evolution by conformational epistasis. Science 2007, 317:1544-1548

44. Lind PA, Berg OG, Andersson DI: Mutational robustness of ribosomal protein genes. Science 2010, 330:825-827.

45. Kristensen DM, Wolf Yl, Mushegian AR, Koonin EV: Computational methods for gene orthology inference. Brief Bioinf 2011.

46. Lind PA, Tobin C, Berg OG, Kurland CG, Andersson DI: Compensatory gene amplification restores fitness after inter-species gene replacements. $\mathrm{Mol}$ Microbiol 2010, 75:1078-1089.

47. Drummond DA, Wilke CO: Mistranslation-induced protein misfolding as a dominant constraint on coding-sequence evolution. Cell 2008, 134(2):341-352.

48. Wolf MY, Wolf YI, Koonin EV: Comparable contributions of structural functional constraints and expression level to the rate of protein sequence evolution. Biol Direct 2008, 3:40.

49. Wolf $Y$, Gopich IV, Lipman DJ, Koonin EV: Relative contributions of intrinsic structural-function constraints and translation rate to the evolution of protein-coding genes. Genome Biol Evol 2010, 2:190-199.

50. Zeng Y, Gu X: Genome factor and gene pleiotropy hypotheses in protein evolution. Biol Direct 2010, 5:37.

51. Mondal UK, Sur S, Bothra AK, Sen A: Comparative analysis of codon usage patterns and identification of predicted highly expressed genes in five Salmonella genomes. Indian J Med Microbiol 2008, 26:313-321.

52. Das S, Ghosh S, Pan A, Dutta C: Compositional variation in bacterial genes and proteins with potential expression level. FEBS Lett 2005, 579:5205-5210.

53. Carbone A, Zinovyev A, Képès F: Codon adaptation index as a measure of dominating codon bias. Bioinformatics 2003, 19:2005-2015.

54. Rocha EP, Danchin A: An analysis of determinants of amino acids substitution rates in bacterial proteins. Mol Biol Evol 2004, 21(1):108-116.

55. Krylov DM, Wolf Yl, Rogozin IB, Koonin EV: Gene loss, protein sequence divergence, gene dispensability, expression level, and interactivity are correlated in eukaryotic evolution. Genome Res 2003, 13(10):2229-2235.

56. Drummond AD, Bloom JD, Adami C, Wilke CO, Arnold FH: Why highly expressed proteins evolve slowly? Proc Natl Acad Sci USA 2005, 102(40):14338-14343.

57. Marsella L, Sirocco F, Trovato A, Seno F, Tosatto SCE: REPETITA: detection and discrimination of the periodicity of protein solenoid repeats by discrete Fourier transform. Bioinformatics 2009, 25:i289-i295.

58. Smole Z, Nikolic N, Supek F, Smuc T, Sbalzarini IF, Krisko A: Proteome sequence features carry signatures of the environmental niche of prokaryotes. BMC Evol Biol 2011, 11:26.

59. Kiraga J, Mackiewicz P, Mackiewicz D, Kowalczuk M, Biecek P, Polak N, Smolarczyk K, Dudek MR, Cebrat S: The relationships between the isoelectric point and: length of proteins, taxonomy and ecology of organisms. BMC Genomics 2007, 8:163.

60. Williams DL, Slayden RA, Amin A, Martinez AN, Pittman TL, Mira A, Mitra A, Nagaraja $V$, Morrison NE, Moraes M, Gillis TP: Implications of high level pseudogene transcription in Mycobacterium leprae. BMC Genomics 2009, 10:397.

61. Gómez-Valero L, Rocha EPC, Latorre A, Silva FJ: Reconstructing the ancestor of Mycobacterium leprae: The dynamics of gene loss and genome reduction. Genome Res 2007, 17(8):1178-1185.

62. Ran L, Larsson J, Vigil-Stenman T, Nylander JAA, Ininbergs K, Zheng WW, Lapidus A, Lowry S, Haselkorn R, Bergman B: Genome erosion in a nitrogen-fixing vertically transmitted endosymbiotic multicellular cyanobacterium. PLoS One 2010, 5(7):e11486.

63. Willenbrock H, Friis C, Friis AS, Ussery DW: An environmental signature for 323 microbial genomes based on codon adaptation indices. Genome Biol 2006, 7(12):R114

doi:10.1186/1745-6150-6-48

Cite this article as: Peralta et al: Sequence variability of Rhizobiales orthologs and relationship with physico-chemical characteristics of proteins. Biology Direct 2011 6:48. 